\title{
On the Study of PEM Fuel Cells by Transmission Electron Microscopy
}

\author{
S. Rasouli ${ }^{1}$, D. Groom ${ }^{1}$, K. Yu ${ }^{1}$, A. Godoy ${ }^{1}$, A. Bovik ${ }^{2}$, D. Myers ${ }^{3}$, Naotoshi Nakashima ${ }^{4}$ and P. J. \\ Ferreira $^{1,3}$ \\ 1. Materials Science and Engineering Program, University of Texas at Austin, Austin, TX \\ 2. Department of Electrical and Computer Engineering, University of Texas at Austin, Austin, TX, \\ 3. Argonne National Laboratory, Argonne, Illinois, United States \\ 4. Department of Applied Chemistry, Kyushu University, Fukuoka, Japan
}

The long-term efficiency of proton exchange membrane fuel cells (PEMFC) is largely restricted by the instability of catalyst nanoparticles during fuel cell operation. Due to their large surface area-tovolume ratio, Pt and Pt-alloy nanoparticles have a strong tendency to grow in size over short time scales, which lead to a reduction in their electrochemically-active surface area, and consequently to an undesired catalyst deactivation and reduction in cell performance after several cycles [1]. Yet, it is still unclear what the main degradation mechanism is, particularly whether modified Ostwald ripening or coalescence is predominant for particle growth within the cathode.

To address this issue, we first performed a post-mortem TEM study to compare the particle size distribution and morphology of the particles at different regions of the cathode side of the PEMFCs before and after voltage cycling. In order to determine the particle size distribution of these PEMFCs rapidly and accurately from TEM images, we developed an image processing protocol based on a combination of computer vision and machine learning techniques (Fig. 1). Furthermore, to better understand the 3-D shape of the NPs and carbon support, as well as measure the interparticle distance, electron tomography of the NPs was performed (Fig. 2).

Voltage cycling of the PEMFCs has been performed under high potential (0.4-1.05 V) and low humidity (30\% RH), which affects modified Ostwald ripening and particle precipitation in the membrane. High temperature $\left(90^{\circ} \mathrm{C}\right)$ cycling was also performed as it influences particle movement and coalescence. The results show that particle growth and particle loss to the membrane is more severe in the high potential sample than in the high temperature, while no significant particle growth and particle precipitation in the membrane can be observed in the low humidity sample (Fig. 3). This parametric study provides an insight into the dominant mechanism of electrochemical active surface area loss. Subsequently, in order to analyze the NP behavior in real time at different stages of fuel cell cycling, a set-up was developed to simulate the effect of voltage cycling on the cathode side of the fuel cell. For this purpose, NPs supported on carbon were deposited on gold TEM grids attached to a gold plate, which were used as a working electrode in a three-electrode electrochemical cell. In this fashion, pre-defined locations of the electrocatalyst on the TEM grid were analyzed before and after cycling using aberration-corrected TEM/STEM. We noticed particle migration, changes of the carbon support structure and Pt re-deposition on NPs. [2]

\section{References:}

[1] Stamenkovic, V. R. et al, Journal of the American Chemical Society 2006, 128, 8813-8819.

[2] Research supported in part by Asst. Sec. for Energy Efficiency and Renewable Energy, Off. of FreedomCAR and Vehicle Technologies, as part of the High Temperature Materials Laboratory User Program, ORNL, managed by UT-Battelle LLC for the U.S. Department of Energy, under contract \# DE-AC05-00OR22725 


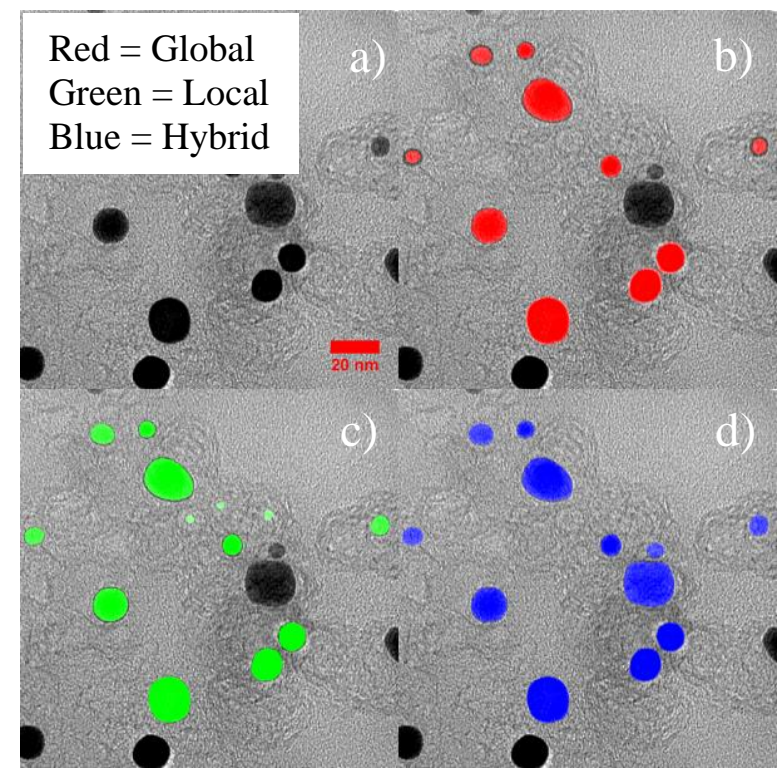

Figure 1. a) Bright-Field TEM image, b) Global thresholding produces two false negatives due to close particles being incorrectly segmented; c) Local thresholding produces three false positives due to regions of the substrate being counted as particles and d) Hybrid thresholding, which produces the best segmentation.

a)

b)
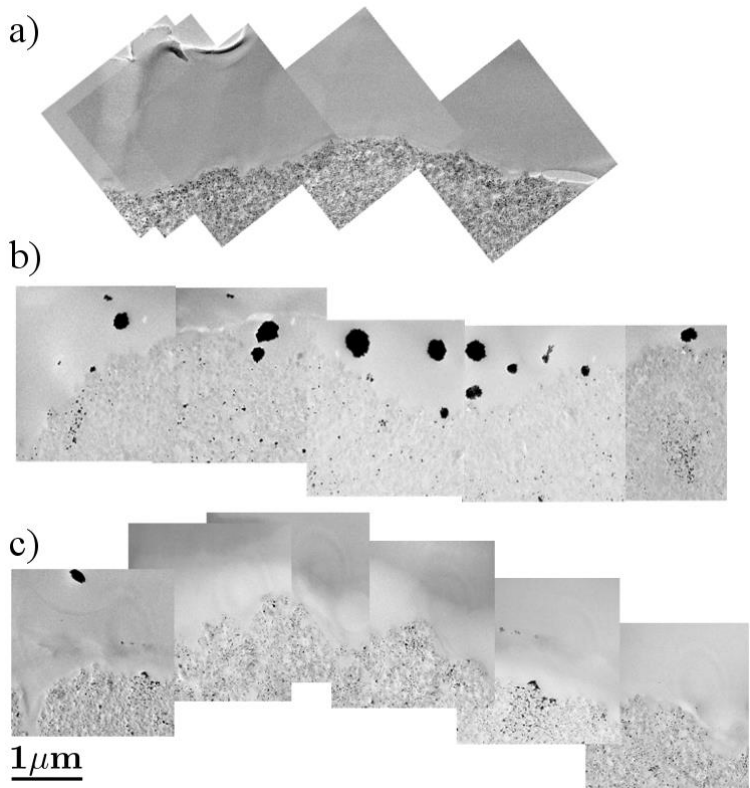

Figure 3. TEM bright-field images of the NPs in the cathode, and the cathode-membrane interface after $10 \mathrm{~K}$ cycling under a) $30 \% \mathrm{RH}$; b) $1.05 \mathrm{~V} \mathrm{c)} 90^{\circ} \mathrm{C}$.

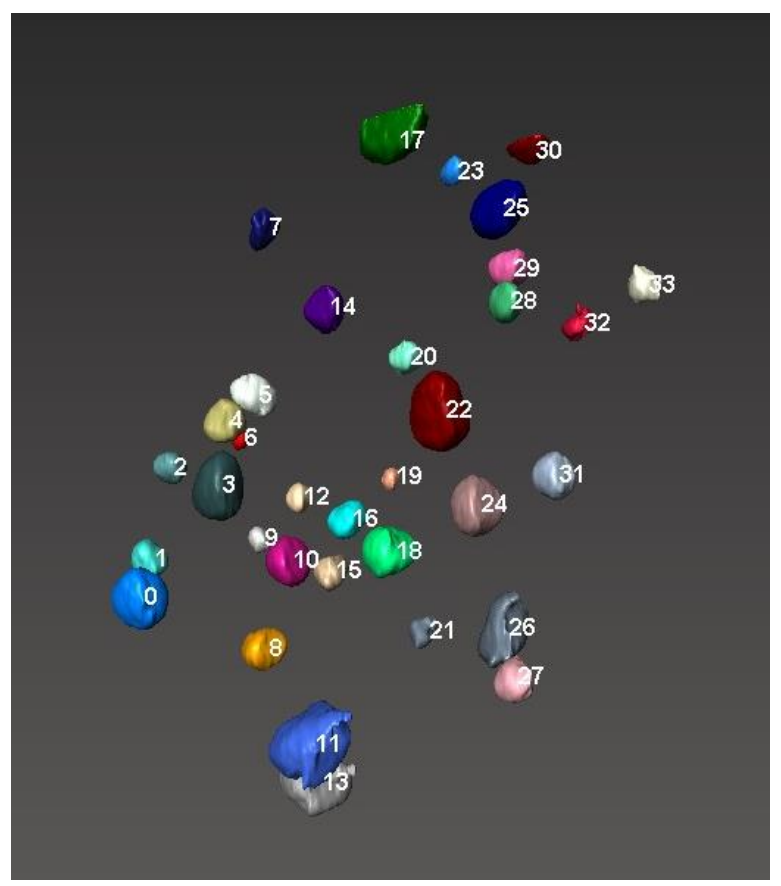

Figure 2. TEM tomography to calculate the 3-D interparticle distance.

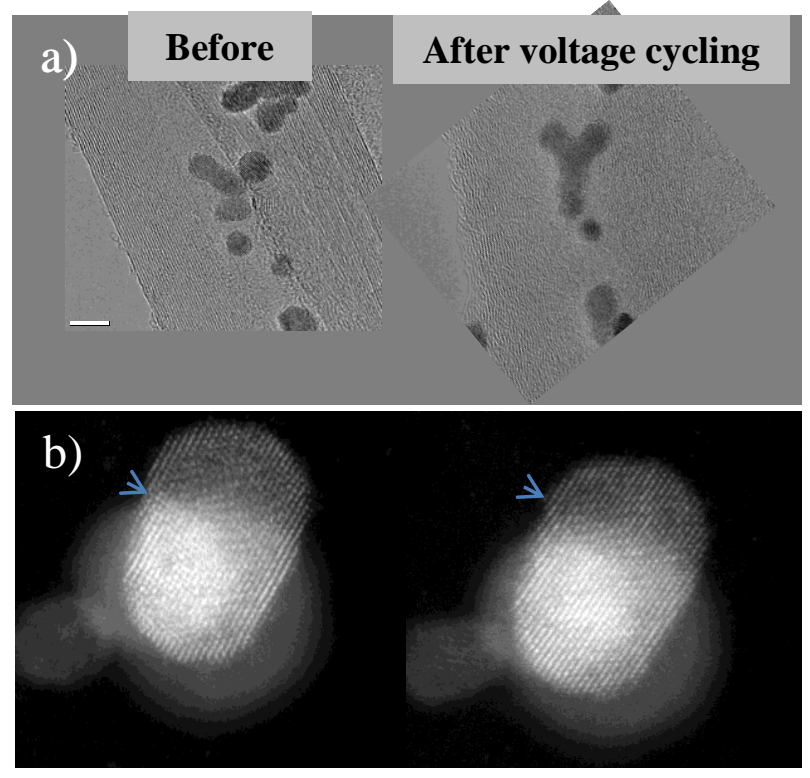

Figure 4. Identical location TEM showing a) the migration of NPs and b) the re-deposition of Pt atoms on a NP. 